\title{
GTR may have no added value over connective tissue grafts for gingival recession defects
}

Danesh-Meyer MJ, Wikesjö UME. Gingival recession defects and guided tissue regeneration: a review. J Periodont Res 2001; 36:341-354

Question: What is the efficacy of guided tissue regeneration (GTR) for recession defects and how does it compare to connective tissue grafts (CTG)?

Objective To summarise the efficacy of GTR for recession defects and compare this with connective tissue grafts (CTG).

Data sources Medline 1985-2000 (search terms not given).

Study selection English-language papers evaluating gingival recession in humans were included. Other criteria were not described and no details of independent evaluation decisions on study eligibility.

Data extraction Tables were constructed separately for GTR resorbable and nonresorbable barriers and for studies comparing GTR with CTG. Although no true meta-analyses were attempted, arithmetic means of outcomes were calculated pooling different study designs together.

Results Thirty studies were identified. From a mainly qualitative assessment, GTR appeared to offer no advantage over CTG with respect to clinical outcomes of root coverage. Technical difficulties of GTR were also highlighted including achieving primary flap closure and membrane exposure. Differences in study design and reporting hampered efforts to formally pool the data.

Conclusion The authors conclude that the use of GTR does not result in greater benefits than CTG alone.

Evidence-Based Dentistry (2002) 3, 103-104. doi:10.1038/ sj.ebd. 6400144

Address for correspondence: Professor UME Wikesjö, Temple University School of Dentistry, Department of Periodontology, 3223 North Broad Street, Philadelphia PA 19140, USA.

\section{Commentary}

Gingival recession can be a problem for patients for many reasons. In patients with a high lip-line, aesthetics may be compromised. Dentinal hypersensitivity can significantly affect a patient's quality of life, by limiting the types of foods they are able to eat. Sensitivity can also interfere with normal hygiene procedures, leading to an increased plaque accumulation and an increased risk of further recession. Danesh-Meyer and Wikesjö reviewed the issue of using GTR for the reconstruction of this gingival defect. This commentary is based on the section of their paper that compares GTR with the current gold standard in root coverage procedures, the CTG.

As with any healthcare intervention, there are risks and benefits to each of the options. CTG have been shown to provide excellent root coverage but there is one major failing: the procedure necessitates the use of two surgical sites, the donor site (often the palate) and the recipient site. It is assumed that patients will be more uncomfortable after surgery as a result. With GTR, especially with the bioresorbable membranes, there is only one surgical site. (Nonresorbable membranes require a second surgical procedure to remove the membrane, and thus they offer no advantage over CTG.) Presumably, having one site would produce less post-operative morbidity. There is, however, a dearth of evidence on any quality-of-life outcomes of GTR. ${ }^{1}$ The most significant drawback to the use of GTR is cost: it is a much more expensive root coverage procedure than CTG (at least in Canada).
The authors of this review paper intended at the outset to undertake a meta-analysis comparing the two techniques. They examined papers from the inception of the use of GTR in a clinical setting to the time of submission of the paper. They stated they could not pool the results of the studies in order to perform the statistical analysis required (ie, meta-analysis). Many of the papers examined used their own 'pet' surgical techniques and biomaterials, which, together with inadequate reporting, precluded pooling of the data.

The authors also stated sample size was a limiting factor. This is not the case with a meta-analysis; in fact, the converse is true. A meta-analysis is a powerful statistical tool, in that data from studies with various (including small) sample sizes are pooled to produce a larger sample. These data 
are then analysed as one study, while controlling for some of the differences between studies.

In a systematic review of the literature, which is sometimes referred to as a meta-analysis although in fact the latter actually describes the statistical methodology, rigorous and explicit methods are employed to search for and critically appraise the entire body of related clinical-research evidence. The search strategy is described so that the entire process is (as all research should be) reproducible. What we have here is a narrative review. The primary problem with such a review is, consciously or not, the author may search the literature in a biased way to support their ideas. As such, it does not permit the reader to check the assumptions of the authors as to how or why they included the studies they did, or to replicate the process. $^{2}$ In addition, the level and quality of the evidence is evaluated and may be taken into account in the statistical process.

Despite these flaws, this paper appears to be a very thorough examination of the literature. Nine clinical trials (two case-control trials and seven randomised controlled trials) were included in the evaluation. From the table of summary results, it is evident there is no advantage of GTR over CTG in any of the clinical parameters. In fact, CTG provide improved root coverage and an increase in the width of keratinised gingiva compared with GTR. The authors speculate that this may be due to exposure of the GTR membranes during initial healing. In fact, regression analysis of the results from nonresorbable membranes found membrane exposure was negatively related with a reduction in recession depth.
Although following the protocol for systematic reviews could have strengthened the results of this paper, the evidence presented is clear-cut. There is no clinical advantage of, and therefore no reason to use, GTR for root coverage procedures.

1. Tatakis $D$, Trombelli L. Adverse effects associated with a bioabsorbable guided tissue regeneration device in the treatment of human gingival recession defects. A clinicopathologic case report. J Periodontol 1999; 70:542-547.

2. Sutherland SE, Matthews DC, Fendrich P. Clinical practice guidelines in dentistry. Part II. By dentists, for dentists. J Can Dent Assoc 2001; 67:448-452.

Debora C Matthews

Division of Periodontics, Faculty of Dentistry, Dalhousie University, Halifax, Nova Scotia, Canada 